\title{
Proposal and Its Evaluation of Neighbors Based Routing for ad hoc networks
}

\author{
Kentaro Aburada \\ Oita National College of Technology, Japan \\ aburada@oita-ct.ac.jp \\ Hisaaki Yamaba, Shinichiro Kubota, Tetsuro Katayama \\ University of Miyazaki, Japan \\ Mirang Park \\ Kanagawa Institute of Technology, Japan \\ Naonobu Okazaki \\ University of Miyazaki, Japan
}

\begin{abstract}
In ad hoc networks, due to the mobility of nodes, communication links are unstable and restricted. As such, an efficient routing protocol is needed in order to solve these problems. In the present paper, we propose a neighbors based routing (NBR) protocol by constructing paths in an area in which a large number of nodes exists. Simulations confirm that the proposed protocol has higher connectivity and a lower control overhead than existing protocols in topologies in which nodes move.
\end{abstract}

Keywords: Ad hoc networks, Routing Protocol, Route Repair, AODV-BR

\section{Introduction}

In recent years, mobile ad hoc networks (MANET) have attracted attention because of the development and popularization of wireless communication technology [1].

Mobile ad hoc networks can be constructed by mobile nodes without a fixed infrastructure. These nodes can send packets directly to each other by wireless communication. Moreover, a node can communicate with other distant nodes, which are outside radio range, by relaying packets. Therefore, MANET is expected to be used as communication tool in emergency situations, for example, at disaster sites, and for data-gathering using sensors. However, due to the mobility of nodes and the limitation of battery capacity, MANET has problems such as route disconnection and a decrease in the packet reception ratio (PRR).

In MANET, various routing protocols are actively being investigated [2]-[5]. It is important to reduce the risk of route disconnections, for example due to increases in power consumption and waiting time, in wireless routing.

Accordingly, methods for repairing disconnected links in

[2] and avoiding an interference region in [4] [5] have been proposed. As an expansion of the ad hoc ondemand distance vector (AODV) [3]), which is the typical routing protocol used in MANET, the AODV$\mathrm{BR}$ (AODV with backup routes) was proposed to repair disconnected links 
[2]. In the AODV-BR, nodes around the constructed route have alternate paths to other nodes on the route, which are used in order to repair disconnected links. When the AODV-BR detects a disconnected link, the node at the link broadcasts data packets. Thus, nodes that receive these packets can restart communication using an alternate path. A method of using a node as position information by GPS as a means of routing instead of flooding was proposed in previous studies [4] and [5]. In [4], PRR x Distance was proposed in order to avoid the interference region based on the packet reception rate (PRR) and the distance to the destination. In addition, in [5], interference-aware energy efficient geographical (IEG) routing was proposed based on the power efficiency of each link. PRR x Distance measures the PRR by packet exchange with neighbor nodes. However, the effectiveness of the PRR decays due to frequent node mobility. Thus, each node must measure PRR repeatedly. Consequently, PRR x Distance cannot immediately adapt to changes in a network. In the IEG, a route that has high power efficiency is constructed by means of measuring the radio field strength instead of the PRR. Therefore, links on the route are shorter than PRR x Distance and, as a result of node mobility, are difficult to disconnect.

In the present paper, we propose a neighbors-based routing (NBR) protocol for easy repair of disconnected routes based on the number of neighbors. The NBR considers neighbors around the route and constructs a route for which alternate paths are easy to use without using position information.

\section{Problems with Existing Protocols}

In the AODV-BR, a route that does not consider neighbor nodes is constructed. Hence, the number of alternate paths is reduced and it is difficult to repair disconnected links. The AODV-BR cannot repair the route flexibly because the disconnected of the alternate path is not considered. In addition, although a route is not constructed in the interference region the route can be constructed around the interference region (Fig. 1). In this case, some of the nodes around the route are in the interference region and may be not available for repairing the route.

In the IEG, each node requires a device that can measure the radio field strength. In addition, since

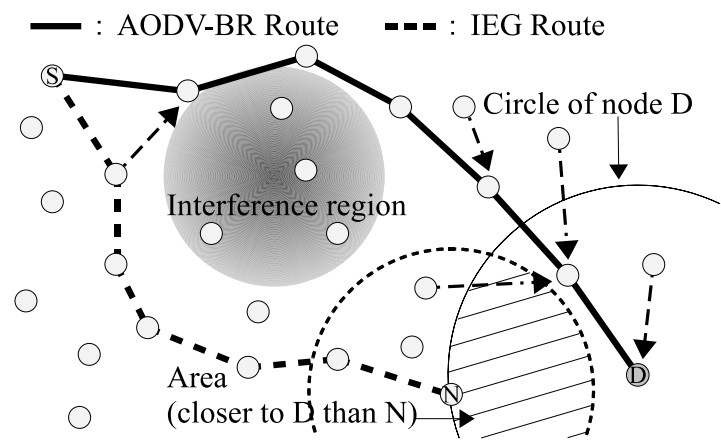

Fig. 1. Problems of existing protocols.

position information is used to choose the next node, the IEG ceases routing in topologies such as that shown in Fig. 1. In Fig.1, as a next hop, node $\mathrm{N}$ must choose a neighbor node that is closer to the destination. These topologies frequently appear upon expansion of the interference region. Once the routing ceases, the IEG switches over to the AODV and resumes communication. However, the AODV has problems with packet loss and power consumption because packets are sent to nodes to which the IEG has already sent an RREQ.

\section{Proposed Method}

In the present paper, we propose an NBR protocol that can easily repair disconnected links by constructing a route based on a number of neighbor nodes and alternative paths.

First, the source node floods RREQs, and each node confirms the state of links with neighbor nodes. The destination node then sends back an RREP upon receiving an RREQ. The RREP is then transmitted to the source node while constructing routes and alternative paths. The source node can receive route information but communicates using the route that has the largest number of neighbor nodes on these routes. In addition, each node can use its route information as an alternative path to repair a disconnected link. Consequently, the NBR can easily repair the disconnected link. Moreover, the NBR introduces the concept of the extension variable in order to increase the number of alternative paths and broaden the route construction area. 


\subsection{Route Evaluation Formula}

In the NBR, alternative paths are recorded in the route table of each node around the route as the AODV-BR. In addition, in the NBR, each node on the route records the paths in the same manner. These paths are the reutilization of received route information. Hence, a significant amount of route information may lead to easier repair. In the NBR, a node that has several neighbor nodes can receive a great deal of route information. Therefore, the evaluation value $\boldsymbol{E}$ of the route can be derived as follows:

$$
\boldsymbol{E}=\frac{N s u m}{h}
$$

where $N_{\text {sum }}$ denotes the sum of the neighbor nodes of each node on a route, and $h$ denotes the number of intermediate nodes between the destination and the node that has received the route information. The source node decides the route that has the highest $\boldsymbol{E}$ using Eq and uses this route for communication. In addition, each node can reduce traffic by relaying only route information that has a higher $\boldsymbol{E}$.

\subsection{Extension Value}

The NBR obtains the hop count from the source node by receiving an RREQ. Each node constructs a route by sending an RREP to a node that has a lower hop count. Thus, an RREP does not reply to a node that has a high hop count. As a result, the NBR cannot construct a route that has several alternative paths, and cannot sufficiently respond to disconnected link. Therefore, the NBR introduces the concept of Extension Variable for transmitting an RREP to a node hat has a high hop count.

In the present paper, $k$ denotes the value of Extension Variable. Here, $k$, which is configured in advance, refers to the number of times that a node can rule out the limit of hop count in the transfer condition of an RREP. The NBR can increase the number of alternative paths and broaden the route construction area.

Fig. 2 shows an example of a route with $k=2$. The route has 20 neighbor nodes and contains five intermediate nodes. Therefore, the evaluation value of the route is 4 . When $k=0$, the route is constructed by under route (Fig.2).

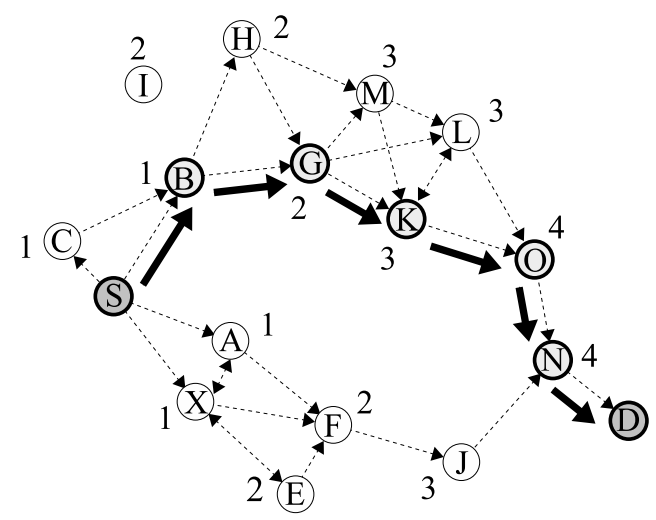

Fig. 2. Example of NBR $(\mathrm{k}=2)$.

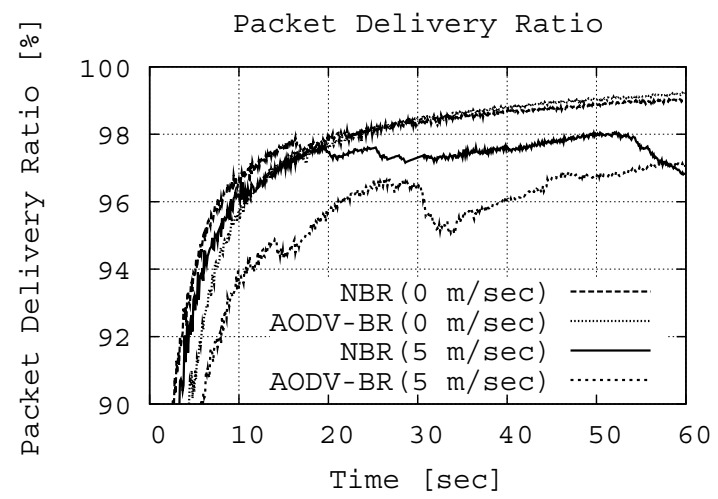

Fig. 3. Packet delivery ratio.

\section{Evaluation}

In order to evaluate the performance of the proposed method NBR, we compare the simulation results of NBR with the traditional AODV-BR method. The simulation measured the packet delivery ratio and the control overhead using Network Simulator-2 (NS-2) [6]. In the simulation, we compare the results for a topology with a moving node to the results for a topology without a moving node. We evaluate the wait time until route construction, the packet delivery ratio, and the control overhead.

\subsection{Simulation Environment}

The proposed simulation modeled a network of 100 mobile nodes placed randomly within a 1,000 meter $\mathrm{x}$ 
1,000 meter area. Two of the nodes were placed at (100, $100)$ and $(900,900)$ as a source node and a destination node, respectively. We used IEEE 802.11 as the medium access control protocol. The radio propagation range for each node was 250 meters, and the channel capacity was $2 \mathrm{Mbps}$. During the simulations, each node moves according to the random waypoint model at a speed of up to $5 \mathrm{~m} / \mathrm{s}$.

The source node transmits data packets to the destination at a rate of five packets per second. The size of the data payload is 1,024 bytes. Each run is executed for a simulation time of 60 seconds. These parameter uses [2] as a reference.

\subsection{Packet delivery ratio}

The packet delivery ratios with NBR and the AODVBR are shown in Fig. 3. Note that both NBR and the AODV-BR have high packet delivery ratios in topologies without node mobility. However, in topologies with moving nodes, the packet delivery ratio of the AODV-BR decreases more than that of NBR. Since NBR constructs several alternate paths, NBR can repair broken links due to moving nodes.

On the other hand, the AODV-BR cannot repair such broken links because the AODV-BR has relatively few alternate paths. Therefore, the individual nodes cannot forward data packets to the destination, and the packet delivery ratio of the AODV-BR decreases in topologies with moving nodes.

\subsection{Control overhead}

Fig.4 shows the control overhead with NBR and the AODV-BR. Both NBR and the AODV-BR have slightly increased control overheads in topologies without moving nodes. However, in topologies with moving nodes, the control overhead of the AODV-BR increases significantly to approximately 120 kbytes. The control overhead of NBR increased but eventually settled at approximately 30 kbytes. The frequency of route reconstruction by the AODV-BR is higher than that for NBR for all simulations. On average, the route reconstruction frequency is 1.2 times per simulation for NBR and 10.2 times per simulation for the AODV-BR. This indicates that the control overhead of the AODV$\mathrm{BR}$ was increased due to the numerous route

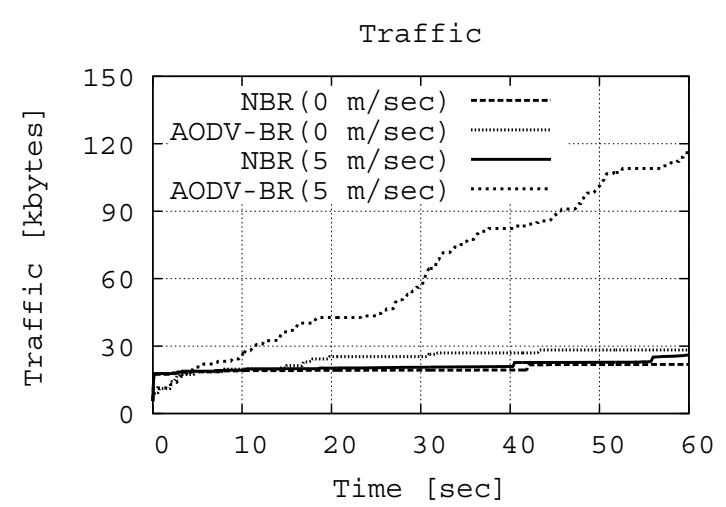

Fig. 4. The control overhead.

reconstructions performed by the AODV-BR.

\section{Conclusion}

We proposed neighbors-based routing (NBR), which constructs alternative paths and routes based on the number of neighbor nodes. Since NBR uses extension variable $\mathrm{k}$ to increase the number of alternative paths and broaden the route construction area, NBR can easily repair disconnected links using alternative paths. In order to evaluate NBR, we compare the packet delivery ratio with the control overhead of NBR and the traditional AODV-BR method using NS-2. The results indicate that

NBR maintains a high packet delivery ratio in topologies with moving nodes, and the present simulations confirmed that the proposed protocol has a higher connectivity and a lower control overhead than existing protocols in topologies with moving nodes.

\section{References}

[1] C-K.Toh.: “Ad Hoc Mobile Wireless Networks: Protocols ans Systems",Prentice Hall (2001).

[2] S-J, Lee. and M, Gerla.: "AODV-BR: Backup Routing in Ad hoc networks", Proc. of IEEE Wireless Communications and Networking Conference, Vol. 3, pp. 1311-1511 (2000).

[3] C, Perkins., E, Belding-Royer. and S, Das.: "Ad hoc on-demand distance vector (AODV) routing", http://www.ietf.org/rfc/rfc3561.txt (2003). 
[4] K, Seada., M, Zuniga., A, Helmy. and B, Krishnamacharim.:”Energy-efficient forwarding strategies for geographic routing in lossy wireless sensor networks", Proc. ACM SenSys, pp. 108-121 (2004).

[5] J, Kim. and Y, Kwon.: "Interference-Aware EnergyEfficient Geographical Routing for IEEE 802.15.4a Networks", IEICE TRANSACTIONS on Communications, Vol. E93-B, No. 4, pp. 1024-1028 (2010).

[6] http://www.isi.edu/nsnam/ns. 